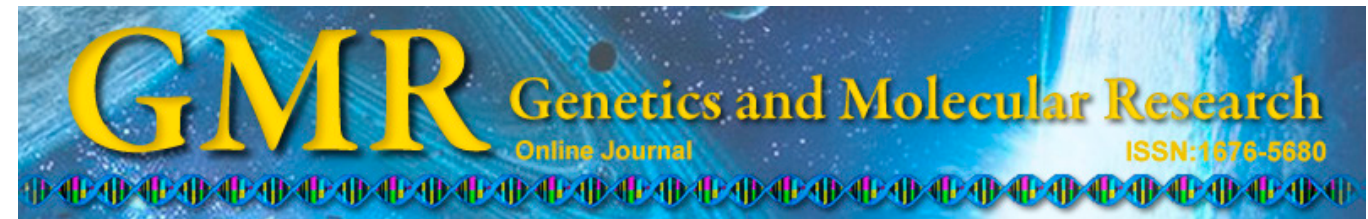

\title{
Hypertension-mediated enhancement of JNK activation in association with endoplasmic reticulum stress in rat model hippocampus with cerebral ischemia-reperfusion
}

\author{
Y.N. Zhao ${ }^{1 *}$, J.M. Li ${ }^{2 *}$, C.X. Chen ${ }^{1}$, P. Zhang ${ }^{1}$ and S.X. Li ${ }^{1}$ \\ ${ }^{1}$ Department of Rehabilitation, \\ North China University of Science and Technology, Tangshan, China \\ ${ }^{2}$ The Affiliated Hospital, North China University of Science and Technology, \\ Tangshan, China \\ *These authors contributed equally to this study. \\ Corresponding author: J.M. Li \\ E-mail: zhaoyan0930@126.com
}

Genet. Mol. Res. 14 (3): 10980-10990 (2015)

Received October 3, 2014

Accepted June 8, 2015

Published September 21, 2015

DOI http://dx.doi.org/10.4238/2015.September.21.10

\begin{abstract}
Acute brain ischemia can induce the activation of c-Jun N-terminal kinases (JNKs). Hypertension is a critical etiology for brain ischemia. We identified the effects of hypertension on the activation of JNK as well as its impact on SP600125, a JNK inhibitor, during endoplasmic reticulum stress (ERS) in the hippocampus using a rat model. Transient whole-brain ischemia was induced by 4-vessel occlusion (bilateral vertebral and bilateral common carotid arteries) in normal and spontaneous hypertensive rats. SP600125 $(0.05 \mathrm{mg} / \mathrm{kg}$, iv) was administered $30 \mathrm{~min}$ before ischemia. Morphological changes in hippocampal nerve cells were observed by cresyl violet staining. Phosphorylation of JNK, and expression levels of CHOP and GPR78, markers for ERS, were detected by western blot at 1, 6, 24, and $48 \mathrm{~h}$, and
\end{abstract}


neurological outcomes were measured using an eight-arm radial maze $48 \mathrm{~h}$ after ischemia. Hypertension apparently aggravated impairment of memory function, decreased the density of surviving neurons, increased phosphorylation of JNK, and enhanced CHOP expression, but reduced GPR78 levels in hippocampal tissues following brain ischemia. SP600125 alleviated neurological dysfunction, improved neuron survival, decreased phosphorylation of JNK and levels of CHOP, but increased expression of GPR78 in rats with hypertension during cerebral ischemia by inhibition of ERS.

Key words: Hypertension; Ischemia/reperfusion; Endoplasmic reticulum stress; c-Jun N-terminal kinases;

CAAT/enhanced I-binding protein

\section{INTRODUCTION}

Hypertension is an independent risk factor for ischemic cerebral stroke and may aggravate nerve injuries after ischemia, thus worsening prognosis (Duverger and MacKenzie, 1988; Nishimura et al., 2000). However, the exact underlying mechanisms remain unclear and require more research.

Mitogen-activated protein kinases (MAPKs) play a critical role in the survival or death of cells. c-Jun N-terminal kinase (JNK), a member of the MAPK family, is activated by a number of stimuli. Activated JNK can phosphorylate a number of downstream substrates, such as MEK and c-Jun, which regulate a number of genes such as TP53 and CASP3 involved in neuronal apoptosis (Park et al., 2000; Manning and Davis, 2003; Tripathi and Sodhi, 2009). The endoplasmic reticulum (ER) is an organelle where macromolecules are synthesized, and calcium ions are stored. The ER is very sensitive to external and internal stimuli and environmental changes inducing accumulation of unfolded proteins and imbalance of calcium homeostasis, a state known as ER stress (ERS). GRP78 and CHOP are proteins that are involved in ERS (Paschen et al., 1998; Rao et al., 2002). Increasing evidence suggests that GRP78 and CHOP may play either a protective or a harmful role after cerebral ischemia in hypertensive animals and humans. Aoki et al. (2001) and Hayashi et al. (2003) have proposed that focal cerebral ischemia-induced severe nerve damage is due to a reduction of GRP78 expression, and that preconditioning may protect the brain against focal cerebral ischemia/ reperfusion (I/R) injuries by downregulating $\mathrm{CHOP}$ expression in the cerebral cortex. There is a relationship between ERS and the activation of the JNK signaling pathway, as evidenced by the fact that transient cerebral ischemia-induced nerve damage is associated with JNK activation and sustained c-Jun expression. Inhibition of ERS by curcumin leads to decreased JNK activity and c-Jun expression, decreasing nerve cell death (Hayashi et al., 2003).

However, there is a lack of data on whether JNK activation influences GRP78 and CHOP expression and whether SP600125, an inhibitor of JNK, can modulate cerebral I/R injuries in the hippocampus in cerebral ischemia combined with hypertension. The present study attempted to answer these questions by using normal and spontaneous hypertensive rats as a model, in an effort to understand the mechanisms involved and the biological consequences of JNK activation in cerebral ischemia under hypertension. 


\section{MATERIAL AND METHODS}

\section{Materials}

Mouse monoclonal antibodies against phosphorylated JNK, CHOP, and GPR78 were obtained from Cell Signaling Biotechnology (Danvers, MA, USA) and Santa Cruz Biotechnology (Santa Cruz, CA, USA). The secondary antibodies used in our experiments were goat anti-mouse IgG obtained from Sigma-Aldrich (St. Louis, MO, USA).

\section{Experimental design}

Normotensive adult male Wistar-Kyoto and spontaneous hypertension male rats (SHR) weighing 250-300 g (Beijing Experimental Animal Center, Chinese Academy of Science, Beijing, China) were selected as a model in this study. Rats were given free access to food and water before surgery. The Wistar-Kyoto rats were divided into two groups: 1) normal rat sham operation (NSO); and 2) normal rat I/R (NI/R). The SHR rats were divided into four groups: 1) hypertensive rat sham operation (SHRSO); 2) hypertensive rat cerebral I/R (SHR+I/R); 3) SHR+I/R+SP600125; and 4) SHR+I/R+dimethyl sulfoxide (DMSO). Normal and hypertensive rats were decapitated at 1, 6, 24, and $48 \mathrm{~h}$ after subjection to I/R for $30 \mathrm{~min}$. The experimental procedures were approved by our local Ethical Committee for animal experiments.

\section{Ischemia induction}

Transient brain ischemia (30 min) was induced by the four-vessel occlusion method, as previously described (Zhang et al., 2003). Under anesthesia using chloral hydrate (300-350 $\mathrm{mg} / \mathrm{kg}, i p$ ), vertebral arteries were electrocauterized and common carotid arteries were exposed. Rats were allowed to recover for $24 \mathrm{~h}$ and fasted overnight. Ischemia was then induced by occluding the common carotid arteries using aneurysm clips. Animals were selected for the present experiment during ischemia as indicated by the following conditions: 1) a completely flat bitemporal electroencephalogram; 2) maintenance of dilated pupils; and 3) absence of corneal reflex. Carotid artery blood flow was restored by releasing the clips. Rectal temperature was maintained at approximately $37^{\circ} \mathrm{C}$ during, and for $2 \mathrm{~h}$ after, ischemia. The sham operation was performed using the same surgical exposure procedures except that the carotid arteries were not occluded.

\section{Drug treatment}

SP600125 was dissolved in 1\% DMSO (200 nmol in $5 \mu$ L DMSO) (Cell Signaling Technology, La Jolla, CA, USA). Drug infusion was performed using a micro-injector in both cerebral ventricles (from the bregma: anteroposterior, $0.8 \mathrm{~mm}$; lateral, $1.5 \mathrm{~mm}$; depth, $3.5 \mathrm{~mm}$ ) as previously described (Wang et al., 2011). A volume of $5 \mu \mathrm{L}$ each was infused over $5 \mathrm{~min}$. SP600125 was given to the SHR rats $30 \mathrm{~min}$ before ischemia and then once every $24 \mathrm{~h}$ for 2 days. Controls received the vehicle DMSO only. 


\section{Histology}

Rats were perfusion-fixed with $4 \%$ paraformaldehyde in $0.1 \mathrm{M}$ sodium phosphate buffer ( $\mathrm{pH}$ 7.4) under anesthesia 1, 6, 24, or $48 \mathrm{~h}$ after I/R. Brains were quickly removed and further fixed with the same fixative at $4^{\circ} \mathrm{C}$ overnight. Post-fixed brains were embedded in paraffin, and cut into $5 \mu \mathrm{m}$ coronal sections using a microtome. Paraffin-embedded brain sections were deparaffinized with xylene and rehydrated using an ethanol gradient $(100 \%$ to $70 \%$ $\mathrm{v} / \mathrm{v}$ ), followed by washing with water. Sections were stained with $0.1 \% \mathrm{w} / \mathrm{v}$ cresyl violet and examined by light microscopy. The number of surviving hippocampal CA1 pyramidal cells with obvious cell membranes, nucleus, and nucleolus was counted as the neuronal density (Wang et al., 2011).

\section{Western blotting analysis}

Rats were decapitated under anesthesia at specific time points from reperfusion after 30-min ischemia. The hippocampal tissue was separated into CA1 and CA3/DG regions and CA1 cells were rapidly frozen in liquid nitrogen. Hippocampus tissues were homogenized in ice-cold homogenization buffer [50 mM 3-(N-morpholino)propansulfonic acid, $\mathrm{pH}$ 7.4, 100 $\mathrm{mM} \mathrm{KCl}, 50 \mathrm{mM} \mathrm{NaF}, 20 \mathrm{mM}$ sodium pyrophosphate, $20 \mathrm{mM} \beta$-phosphoglycerol, $320 \mathrm{mM}$ sucrose, $0.2 \mathrm{mM}$ dithiothreitol, $1 \mathrm{mM}$ edetic acid, $1 \mathrm{mM}$ egtazic acid, $0.5 \mathrm{mM} \mathrm{MgCl}, 1 \mathrm{mM}$ $\mathrm{Na}_{3} \mathrm{VO}_{4}, 1 \mathrm{mM}$ p-nitrophenyl phosphate, $5 \mu \mathrm{g} / \mathrm{mL}$ each of aprotinin, leupeptin, and pepstatin A, $1 \mathrm{mM}$ phenylmethylsulfonyl fluoride, and $1 \mathrm{mM}$ benzamidine]. Homogenates were then centrifuged at $800 \mathrm{~g}$ for $15 \mathrm{~min}$ at $4^{\circ} \mathrm{C}$. Supernatants were collected and protein concentrations were determined by the Lowry method. Samples were stored at $-80^{\circ} \mathrm{C}$ until required. Samples (20 $\mu$ g protein each) were separated by 10 or $7.5 \%$ sodium dodecyl sulfate polyacrylamide gel electrophoresis and electrotransferred onto nitrocellulose membranes (Hayashi et al., 2003). After blocking with $3 \%$ bovine serum albumin for $3 \mathrm{~h}$, membranes were probed with the proper primary antibody at $4{ }^{\circ} \mathrm{C}$ overnight. Bound antibodies were detected using alkaline phosphatase-conjugated goat anti-mouse IgG for $2 \mathrm{~h}$. Immunoreactivities were detected using an NBT/BCIP assay kit, according to the manufacturer instructions (Sigma-Aldrich, St. Louis). Protein bands were scanned and analyzed with an image analyzer (LabWorks Software, UVP Inc., Upland, CA, USA).

\section{Spatial memory in the radial arm maze apparatus}

The radial maze was made from gray polyvinylchloride and consisted of eight arms (length: $32 \mathrm{~cm}$, width: $8 \mathrm{~cm}$, height: $19 \mathrm{~cm}$ ) extending radically from an octagonal central area (20 cm across). Rats were trained to the radial maze for two consecutive days with two trials per day. Each rat was placed in the central area of the maze with all arm entries closed. After 10 $\mathrm{s}$, the doors were opened and the rat was permitted to enter any of the eight arms. Only three of the eight arms contained water. The rationale for using three instead of four baited arms was to increase the sensitivity of the task in measuring reference memory errors (RME) by decreasing the probability of making a correct choice by chance. The three arms containing water were randomly determined for each rat and were unchanged over the 2-day learning period. A trial was terminated after either all baits were consumed or after $10 \mathrm{~min}$, whichever occurred first. 
Forty-eight hours after injury, the memory function was assessed using a previously described method (Hayashi et al., 2003). An experimenter who was blinded to the experimental groups scored each rat based on: 1) RME: entering into an arm that was never baited; and 2) working memory errors (WME): re-entering into an arm already visited.

\section{Statistical analysis}

Values are reported as means $\pm \mathrm{SD}$, from five independent rats. Statistical analysis was performed by one-way analysis of variance, followed by Duncan's new multiple range method, using the SPSS statistical package for social science (SPSS Inc., Chicago, IL, USA). $P$ values $<0.05$ were considered to be significant.

\section{RESULTS}

\section{Neuronal cell viabilities in hippocampal CA1 regions}

Cresyl violet staining was used to determine the survival of CA1 pyramidal neurons. Normal cells showed round and weakly stained nuclei. Shrunken cells with pyknotic nuclei after I/R were counted as dead cells. Transient brain ischemia followed by reperfusion induced severe cell death at 1, 6,24, and $48 \mathrm{~h}$ after reperfusion. Compared with brain ischemia in normal rats, hypertension-aggravated cell death and neuron survival were markedly decreased in the SHR $+\mathrm{I} / \mathrm{R}$ group. Pretreatment with SP600125 before ischemia significantly decreased neuronal degeneration and increased neuron survival in the SHR $+\mathrm{I} / \mathrm{R}+\mathrm{SP} 600125$ group compared with the SHR+I/R group. DMSO had no effect on neuronal survival (Figure 1 and Table 1).


Figure 1. Representative photomicrographs of cresyl violet-stained sections of the hippocampus in rats at $24 \mathrm{~h}$ after subjection to ischemia/reperfusion (I/R). A. Normal rats subjected to sham operation. B. Normal rats subjected to I/R. C. Spontaneous hypertension rats subjected to sham operation. D. Spontaneous hypertension rats subjected to I/R. E. Spontaneous hypertension rats subjected to I/R plus dimethyl sulfoxide vehicle; and F. spontaneous hypertension rats with I/R plus SP600125. Magnification 400X. 
Table 1. Quantitative analysis of neuronal survival in the hippocampus in normal and hypertensive rats subjected to ischemia/reperfusion (I/R).

\begin{tabular}{lcccc}
\hline Groups & \multicolumn{4}{c}{ Neuronal density in CA1 region } \\
\cline { 2 - 5 } Times after I/R & $1 \mathrm{~h}$ & $6 \mathrm{~h}$ & $24 \mathrm{~h}$ & $180.10 \pm 20.05$ \\
\hline NSO & $188.65 \pm 20.90$ & $189.8 \pm 19.85$ & $48.80 \pm 19.90$ \\
NI/R & $120.80 \pm 16.75$ & $100.75 \pm 12.50$ & $65.90 \pm 10.75$ & $40.80 \pm 9.75$ \\
SHRSO & $170.10 \pm 19.15$ & $171.24 \pm 18.80$ & $172.10 \pm 18.05$ & $172.50 \pm 18.40$ \\
SHR+I/R & $80.50 \pm 12.48^{\mathrm{a}}$ & $60.75 \pm 10.50^{\mathrm{a}}$ & $38.75 \pm 8.70^{\mathrm{a}}$ & $26.70 \pm 6.10^{\mathrm{a}}$ \\
SHR+I/R+DMSO & $81.45 \pm 12.45$ & $59.80 \pm 10.45$ & $39.25 \pm 8.60$ & $27.20 \pm 6.25$ \\
SHR+I/R+SP600125 & $112.65 \pm 18.50^{\mathrm{b}}$ & $96.80 \pm 11.65^{\mathrm{b}}$ & $80.60 \pm 11.80^{\mathrm{b}}$ & $78.75 \pm 10.80^{\mathrm{b}}$ \\
\hline
\end{tabular}

$\mathrm{NSO}=$ normal rats subjected to sham operation; NI/R $=$ normal rats subjected to $\mathrm{I} / \mathrm{R} ; \mathrm{SHRSO}=$ spontaneous hypertensive rats subjected to sham operation; $\mathrm{SHR}+\mathrm{I} / \mathrm{R}=$ spontaneous hypertensive rats subjected to $\mathrm{I} / \mathrm{R} ; \mathrm{SHR}+\mathrm{I} /$ $\mathrm{R}+\mathrm{DMSO}=$ spontaneous hypertensive rats subjected to I/R plus dimethyl sulfoxide (DMSO) vehicle; SHR $+\mathrm{I} /$ $\mathrm{R}+\mathrm{SP} 600125=$ spontaneous hypertensive rats subjected to I/R plus SP600125. Data are reported as means \pm SD from five rats. ${ }^{\mathrm{a}} \mathrm{P}<0.05$ vs $\mathrm{NI} / \mathrm{R}$; ${ }^{\mathrm{b}} \mathrm{P}<0.05$ vs $\mathrm{SHR}+\mathrm{I} / \mathrm{R}+\mathrm{DMSO}$.

\section{Activation of JNK after I/R}

To assess the JNK activation after brain ischemia under hypertension or treatment with SP600125, we examined levels of phospho-JNK at 1, 6, 24, and $48 \mathrm{~h}$ after I/R. The level of phospho-JNK increased rapidly in the NI/R group compared with the NSO group after $1 \mathrm{~h}$, peaked at $6 \mathrm{~h}$, and decreased after $24 \mathrm{~h}$ before returning to the control level after $48 \mathrm{~h}$. JNK activation was strong at all time points in the SHR $+\mathrm{I} / \mathrm{R}$ group; it steadily increased to a maximum level at $24 \mathrm{~h}$ and slightly decreased $48 \mathrm{~h}$ after reperfusion in comparison with the SHRSO group. Therefore, hypertension enhanced ischemia-induced JNK activation. The use of SP600125 blocked JNK phosphorylation induced by brain ischemia under hypertension in comparison with the DMSO-treated control group (Figure 2).

\section{GPR78 and CHOP expressions during ERS following I/R}

To explore ERS in the hippocampus after brain ischemia under hypertension in the rat model, tissue expression levels of CHOP and GPR78, markers for the ERS, were identified at various time points after I/R. The levels of both GPR78 and CHOP increased rapidly after $1 \mathrm{~h}$, peaked at $24 \mathrm{~h}$, and then decreased after $48 \mathrm{~h}$ following I/R in the normal rat group (NI/R compared with NSO). Although hypertensive rats seemed to have high basal levels of GPR78 and CHOP (SHRSO compared with NSO), they were able to rapidly respond to I/R by increasing these proteins to maximum levels at $6 \mathrm{~h}$ for GPR78 and $24 \mathrm{~h}$ for CHOP, then reducing their expression levels to below the control level (SHRI/R compared with SHRSO) suggesting strong ERS occurring in the hippocampus. Pretreatment with SP600125 apparently resulted in different responses for GPR78 and CHOP in hypertensive rats under I/R conditions: GPR78 was upregulated while CHOP was downregulated (SHR+I/R+SP600125 compared with SHR $+\mathrm{I} / \mathrm{R})$. Note that the DMSO vehicle had no effect on GPR78 and CHOP expression levels (SHRI/R+DMSO compared with SHRI/R) (Figure 3A and B).

\section{Memory function after $\mathbf{I} / \mathrm{R}$}

The results for RME and WME obtained from the memory trials are shown in Table 2. Memory damage was induced in normal rats $48 \mathrm{~h}$ after I/R (NRI/R compared with NRSO). 
Marked increases in the number of RME and WME in the SHR+I/R group suggest that hypertension apparently aggravated memory impairment induced by $\mathrm{I} / \mathrm{R}(\mathrm{SHR}+\mathrm{I} / \mathrm{R}$ compared with $\mathrm{NR}+\mathrm{I} / \mathrm{R}$ ). Marked decreases in the number of RME and WME in the SHR+I/R+SP600125 group suggest that pretreatment with SP600125 before ischemia significantly improved recovery of memory functions in hypertensive rats in response to I/R. DMSO had no effect on the RME or WME in the memory trial.

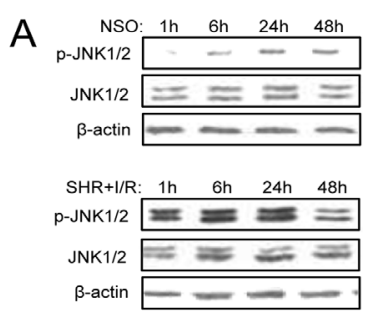

B

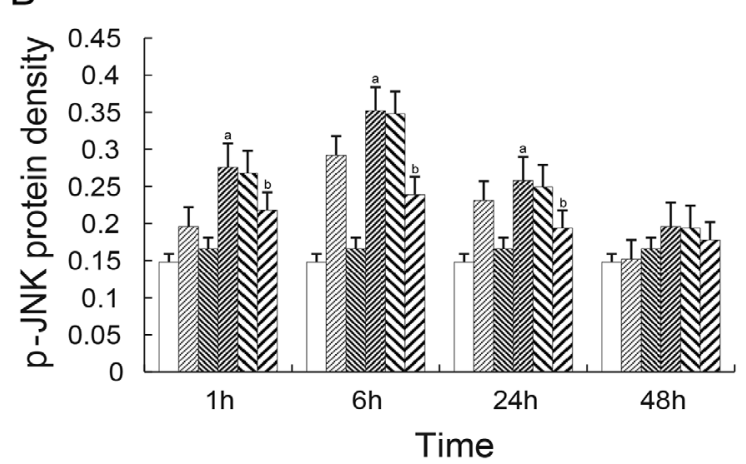

C

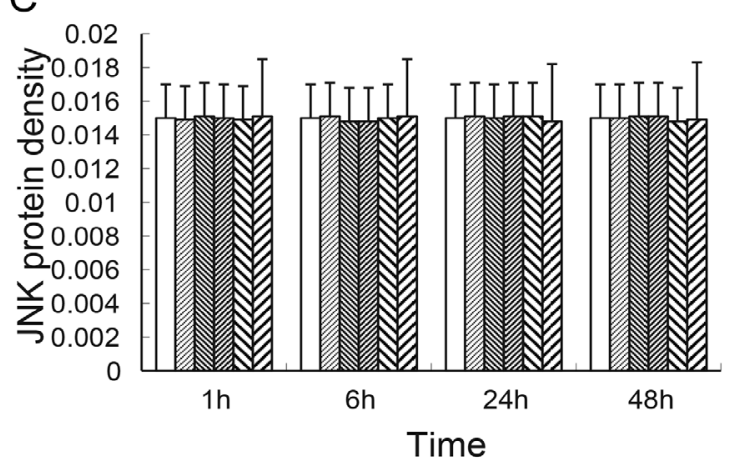

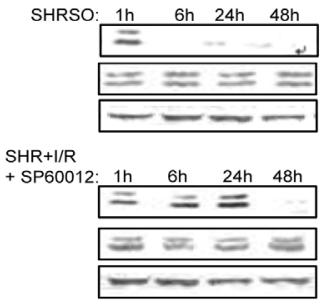

$\square$ NSO group NI/R group SHRSO group SHR+l/R group $\checkmark$ SHR+l/R+DMSO group $\triangle$ SP600125 group

aNSO group ఐNI/R group ه SHRSO group ๑SHR+I/R group $\Delta S H R+1 / R+D M S O$ group 口SP600125 group

Figure 2. c-Jun N-terminal kinase (JNK) activation in the hippocampus in normal and spontaneous hypertension rats subjected to ischemia/reperfusion $(\mathrm{I} / \mathrm{R})$. $\mathrm{NSO}=$ normal rats subjected to sham operation; $\mathrm{NI} / \mathrm{R}=$ normal rats subjected to I/R; SHRSO = spontaneous hypertension rats subjected to sham operation; $\mathrm{SHR}+\mathrm{I} / \mathrm{R}=$ spontaneous hypertension rats subjected to $\mathrm{I} / \mathrm{R} ; \mathrm{SHR}+\mathrm{I} / \mathrm{R}+\mathrm{DMSO}=$ spontaneous hypertension rats subjected to $\mathrm{I} / \mathrm{R}$ plus dimethyl sulfoxide (DMSO) vehicle; SHR+I/R+SP600125 = spontaneous hypertension rats subjected to I/R plus SP600125. A.-C. p-JNK (phospho-JNK), JNK, and $\beta$-actin expression levels were examined by immunoblotting analysis. B. and C. p-JNK, JNK, and $\beta$-actin were scanned and the intensities were normalized to $\beta$-actin. Data are reported as means $\pm \mathrm{SD}$ from five rats. ${ }^{a} \mathrm{P}<0.05$ vs $\mathrm{NI} / \mathrm{R}$; ${ }^{\circ} \mathrm{P}<0.05$ vs $\mathrm{SHR}+\mathrm{I} / \mathrm{R}+\mathrm{DMSO}$. 


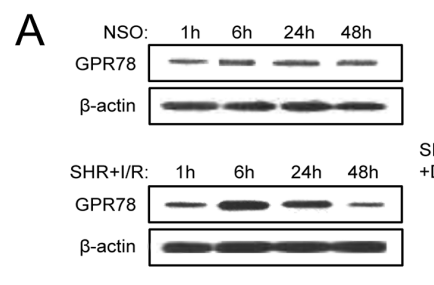

B

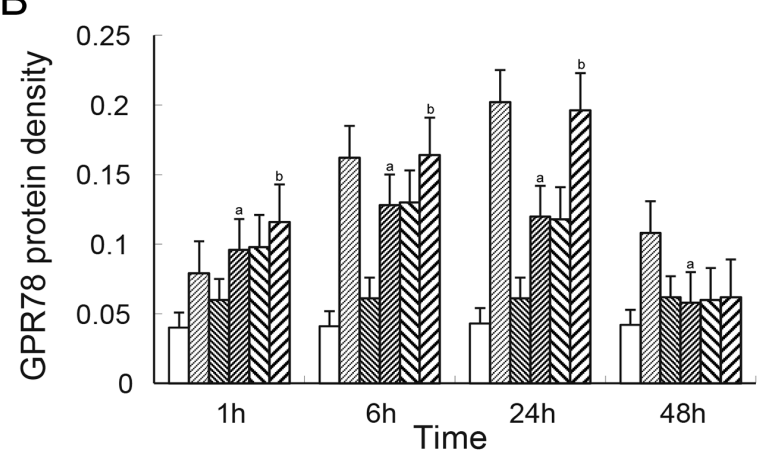

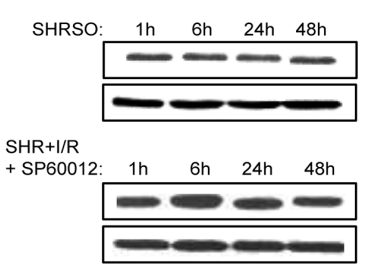

aNSO group

$\square \mathrm{NI} / \mathrm{R}$ group

هSHRSO group

- $S H R+1 / R$ group

వ $S H R+1 / R+D M S O$ group

घSP600125 group
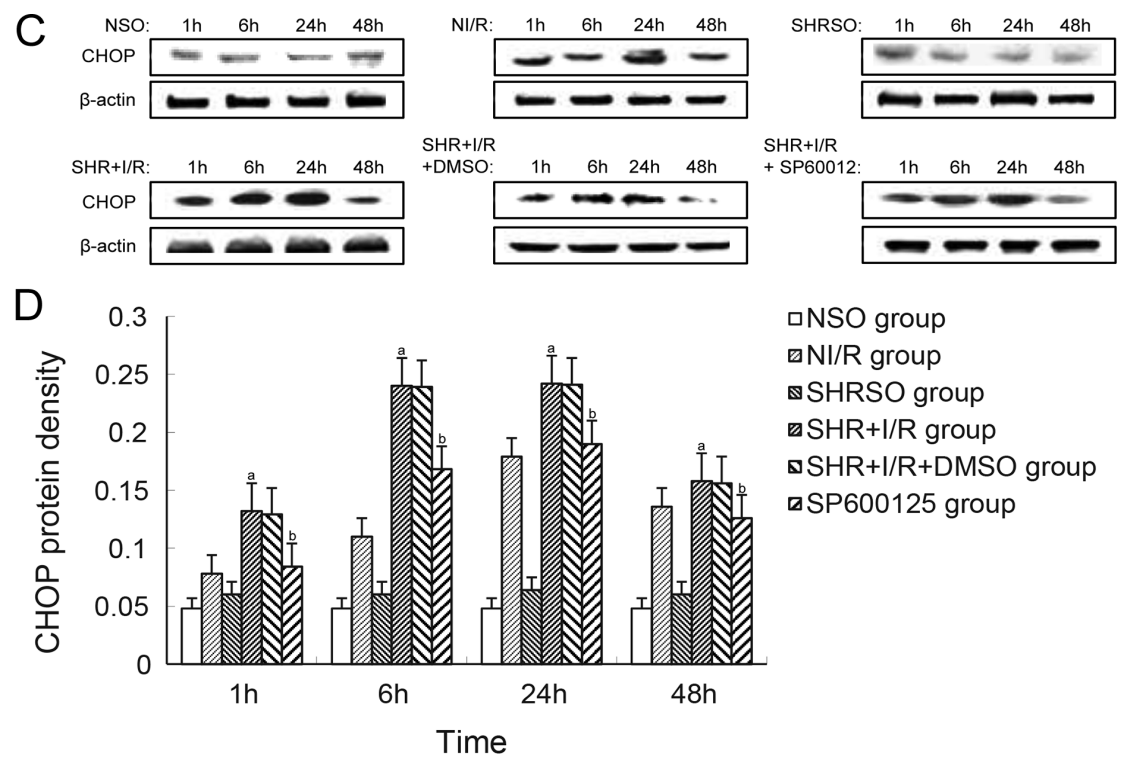

aNSO group

$\square N I / R$ group

$\triangle$ SHRSO group

$\triangle \mathrm{SHR}+\mathrm{I} / \mathrm{R}$ group

వSHR+I/R+DMSO group

هSP600125 group

Figure 3. CHOP and GPR78 expression levels in the hippocampus in normal and spontaneous hypertension rats subjected to ischemia/reperfusion $(\mathrm{I} / \mathrm{R})$. $\mathrm{NSO}=$ normal rats subjected to sham operation; $\mathrm{NI} / \mathrm{R}$ : normal rats subjected to $\mathrm{I} / \mathrm{R}$; SHRSO = spontaneous hypertension rats subjected to sham operation; $\mathrm{SHR}+\mathrm{I} / \mathrm{R}=$ spontaneous hypertension rats subjected to $\mathrm{I} / \mathrm{R} ; \mathrm{SHR}+\mathrm{I} / \mathrm{R}+\mathrm{DMSO}=$ spontaneous hypertension rats subjected to $\mathrm{I} / \mathrm{R}$ plus dimethyl sulfoxide $(\mathrm{DMSO})$ vehicle; SHR $+\mathrm{I} / \mathrm{R}+\mathrm{SP} 600125=$ spontaneous hypertension rats subjected to $\mathrm{I} / \mathrm{R}$ plus SP600125. A.-D. GPR78 (A and B) and CHOP (C and D) expression levels were examined by immunoblotting analysis. B. and D. GPR78, CHOP, and $\beta$-actin were scanned and the intensities were normalized to $\beta$-actin. Data are reported as means $\pm \mathrm{SD}$ from five rats. ${ }^{\mathrm{a}} \mathrm{P}<0.05 v s \mathrm{NI} / \mathrm{R} ;{ }^{\mathrm{b}} \mathrm{P}<0.05$ vs $\mathrm{SHR}+\mathrm{I} / \mathrm{R}+\mathrm{DMSO}$. 
Table 2. Quantitative analysis of memory injuries in normal and hypertensive rats at $48 \mathrm{~h}$ after subjection to ischemia/reperfusion (I/R).

\begin{tabular}{lcc}
\hline Group & RME & WME (error times) \\
\hline NSO & $2.03 \pm 1.26$ & $1.28 \pm 0.45$ \\
NI/R & $5.37 \pm 1.64$ & $3.01 \pm 1.40$ \\
SHRSO & $3.26 \pm 1.38$ & $1.85 \pm 0.80$ \\
SHR+I/R & $7.35 \pm 2.80^{\mathrm{a}}$ & $5.85 \pm 2.10^{\mathrm{a}}$ \\
SHR+I/R+DMSO & $7.40 \pm 2.75$ & $5.90 \pm 2.12$ \\
SHR+I/R+SP600125 & $5.12 \pm 1.56^{\mathrm{b}}$ & $2.86 \pm 1.24^{\mathrm{b}}$ \\
\hline
\end{tabular}

$\mathrm{RME}=$ reference memory errors; $\mathrm{WME}=$ working memory errors; $\mathrm{NSO}=$ normal rats subjected to sham operation; $\mathrm{NI} / \mathrm{R}=$ normal rats subjected to I/R; SHRSO = spontaneous hypertension rats subjected to sham operation; SHR $+\mathrm{I} / \mathrm{R}$ $=$ spontaneous hypertension rats subjected to $\mathrm{I} / \mathrm{R} ; \mathrm{SHR}+\mathrm{I} / \mathrm{R}+\mathrm{DMSO}=$ spontaneous hypertension rats subjected to $\mathrm{I} / \mathrm{R}$ plus dimethyl sulfoxide (DMSO) vehicle; $\mathrm{SHR}+\mathrm{I} / \mathrm{R}+\mathrm{SP} 600125$ = spontaneous hypertension rats subjected to $\mathrm{I} / \mathrm{R}$ plus $\mathrm{SP} 600125$. Data are reported as means $\pm \mathrm{SD}$ from six rats. ${ }^{\mathrm{P}} \mathrm{P}<0.05$ vs $\mathrm{NI} / \mathrm{R}$; ${ }^{\mathrm{b}} \mathrm{P}<0.05$ vs $\mathrm{SHR}+\mathrm{I} / \mathrm{R}+\mathrm{DMSO}$.

\section{DISCUSSION}

As reported, activation of JNK is involved in brain damage induced by hypertension and cerebral I/R (Sugino et al., 2000; Li et al., 2009; Hu et al., 2012; Ormiston et al., 2013). Activation of JNK enhances phosphorylation of c-Fos and c-Jun and facilitates AP-1 formation, which further triggers caspase-3-dependent or -independent signals, implicated in neuron death and memory loss (Tang et al., 2002; Guan et al., 2006). Thus, JNK is an early target and effector for ischemia-induced neuronal damage. In this study, we observed the JNK status in normal and hypertensive rats in response to I/R and explored the modulation effects of SP600125, an inhibitor of JNK, on JNK-mediated phenotype changes. Our results indicate that hypertension exacerbates I/R-induced structural and memory damage to the hippocampus, as proved by the activation of JNK. SP600125 displayed neuro-protective effects mediated by inhibition of ERS.

Ischemia-elicited ERS may be a critical mechanism for JNK signal activation (Liu et al., 2008; Li et al., 2009). Our results showed that in response to I/R, hypertensive rats exhibited an increase in GRP78 from 1 to $6 \mathrm{~h}$ followed by a marked decrease from 6 to $48 \mathrm{~h}$, and increases in CHOP and phospho-JNK from 1 to $24 \mathrm{~h}$ followed by decreases from 24 to $48 \mathrm{~h}$. Thus, a decrease in GRP78 was accompanied by an increase in CHOP in association with an increase in the level of phospho-JNK $24 \mathrm{~h}$ after I/R in hypertensive rats. Decreased GRP78 in extensive ERS increases the accumulation of free inositol-requiring enzyme 1 (IRE1), activates PKR-like endoplasmic reticulum kinase and apoptosis signal regulation kinase IRE1/ TRAF-2/ASK-1, and enhances phosphorylation of JNK and upregulation of CHOP, leading to the loss of nerve cells (Urano et al., 2000). Alleviation of ERS decreased phosphorylation of JNK and death of myocardial cells in a myocardial I/R model (Liu et al., 2008). Preconditioning to ERS inhibited the expression of phosphorylated JNK and decreased neural cell death in the hippocampus in a focal brain ischemia model (Xu et al., 2012). SP600125 reversibly competes for ATP, suppressing JNK, which is followed by downstream inhibition of the generation of phosphorylated c-Jun, inflammatory factor epoxidase-2, interleukin-2, immunoreactivity fiber, tumor necrosis factor, and oxygen-based free radicals (Bennett et al., 2001; Kim et al., 2008). In addition, a "network communication" exists between JNK and a variety of signal pathways, such as the ERK and AKT pathways (Hu et al., 2004; Hong and Kim, 2007). Thus, in hypertensive rats subjected to cerebral I/R, SP600125 inhibition of JNK activation, promo- 
tion of neuron survival, and improvement of memory functions by stabilization of cellular ER may be collectively achieved by reducing inflammatory responses, antagonizing oxidative stress, and balancing "network communication".

ERS is an adaptive response. Moderate ERS is marked by an increase in the levels of GRP78 and calreticulin, which enhance a cell's ability to activate the unfolded protein response, and promote ERS recovery. However, severe or continuous ERS can stimulate the activation of CHOP and caspase-12, and may trigger ERS-related apoptotic pathways, leading to cell death and tissue injury (Aoki et al., 2001; Nieto-Miguel et al., 2007). Tajiri et al. (2004) detected the CHOP protein in the hippocampus and striatum of wild-type and CHOP-/- mice after bilateral common carotid artery ligature for $15 \mathrm{~min}$. The CHOP levels were related to the number of apoptotic cells at $48 \mathrm{~h}$ after reperfusion. The number of dead nerve cells in the hippocampus and striatum was markedly decreased in homozygous Chop knockout mice. Lehotský et al. (2009) reported that the apoptosis rate and CHOP and caspase-12 expression levels decreased while GRP78 increased at each time point in rats after a 10-min ischemia. In our experiments, GRP78 and CHOP expression levels exhibited a different time course to reach maximum levels in hypertensive rats in response to $\mathrm{I} / \mathrm{R}$. As indicated, two peak time points were differentiated at about $18 \mathrm{~h}$. More interesting, GRP78 and CHOP also displayed different responses to SP600125, a JNK inhibitor. GRP78 expression was enhanced but CHOP expression inhibited in hypertensive rats treated with $\mathrm{I} / \mathrm{R}$. The possible explanation is that under hypertension I/R may further increase cytotoxic factors, such as glutamic acid, calcium overload, and oxygen radicals (Hirata et al., 1995; Srinivasan and Sharma, 2012) inducing severe ERS, as evinced by increased CHOP and decreased GRP78 levels, resulting in nerve cell death and a severe memory loss.

In summary, during cerebral ischemia hypertension enhanced activation of JNK and upregulation of CHOP, but inhibited expression of GPR78 in association with severe neuronal cell death in the hippocampus and memory loss. SP600125 protects against I/R injuries in hypertensive rats by inhibition of ERS. These findings provide a new insight into the role of JNK and yield promising therapeutic options for hypertensive patients with stroke.

\section{Conflicts of interest}

The authors declare no conflict of interest.

\section{REFERENCES}

Aoki M, Tamatani M, Taniguchi M, Yamaguchi A, et al. (2001). Hypothermic treatment restores glucose regulated protein 78 (GRP78) expression in ischemic brain. Mol. Brain Res. 95: 117-128.

Bennett BL, Sasaki DT, Murray BW, O’Leary EC, et al. (2001). SP600125, an anthrapyrazolone inhibitor of Jun N-terminal kinase. Proc. Natl. Acad. Sci. U. S. A. 98: 13681-13686.

Duverger D and MacKenzie ET (1988). The quantification of cerebral infarction following focal ischemia in the rat: influence of strain, arterial pressure, blood glucose concentration, and age. J. Cereb. Blood Flow Metab. 8: 449-461.

Guan QH, Pei DS, Xu TL and Zhang GY (2006). Brain ischemia/reperfusion-induced expression of DP5 and its interaction with Bcl-2, thus freeing Bax from Bcl-2/Bax dimmers are mediated by c-Jun N-terminal kinase (JNK) pathway. Neurosci. Lett. 393: 226-230.

Hayashi T, Saito A, Okuno S, Ferrand-Drake M, et al. (2003). Induction of GRP78 by ischemic preconditioning reduces endoplasmic reticulum stress and prevents delayed neuronal cell death. J. Cereb. Blood Flow Metab. 23: 949-961.

Hirata H, Asanuma M, Tanaka K, Kondo Y, et al. (1995). M1 receptors in blood pressure-controlled ischemic spontaneously hypertensive rats. Stroke 26: 1268-1272. 
Hong HY and Kim BC (2007). Mixed lineage kinase 3 connects reactive oxygen species to c-Jun NH2-terminal kinaseinduced mitochondrial apoptosis in genipin-treated PC3 human prostate cancer cells. Biochem. Biophys. Res. Commun. 362: 307-312.

Hu J, Luo CX, Chu WH, Shan YA, et al. (2012). 20-Hydroxyecdysone protects against oxidative stress-induced neuronal injury by scavenging free radicals and modulating NF-אB and JNK pathways. PLoS One 7: e50764.

Hu P, Han Z, Couvillon AD and Exton JH (2004). Critical role of endogenous Akt/IAPs and MEK1/ERK pathways in counteracting endoplasmic reticulum stress-induced cell death. J. Biol. Chem. 279: 49420-49429.

Kim EM, Yang HS, Kang SW, Ho JN, et al. (2008). Amplification of the gamma-irradiation-induced cell death pathway by reactive oxygen species in human U937 cells. Cell Signal 20: 916-924.

Lehotský J, Urban P, Pavlíková M, Tatarková Z, et al. (2009). Molecular mechanisms leading to neuroprotection/ischemic tolerance: effect of preconditioning on the stress reaction of endoplasmic reticulum. Cell Mol. Neurobiol. 29: 917925.

Li LM, Liu QH, Qiao JT and Zhang C (2009). A beta(31-35)-induced neuronal apoptosis is mediated by JNK-dependent extrinsic apoptosis pathway. Neurosci. Bull. 25: 361-366.

Liu XH, Zhang ZY, Sun S and Wu XD (2008). Ischemic postconditioning protects myocardium from ischemia/reperfusion injury through attenuating endoplasmic reticulum stress. Shock 30: 422-427.

Manning AM and Davis RJ (2003). Targeting JNK for therapeutic benefit: from junk to gold? Nat. Rev. Drug Discov. 2: 554-565.

Nieto-Miguel T, Fonteriz RI, Vay L, Gajate C, et al. (2007). Endoplasmic reticulum stress in the proapoptotic action of edelfosine in solid tumor cells. Cancer Res. 67: 10368-10378.

Nishimura Y, Ito T and Saavedra JM (2000). Angiotensin II AT(1) blockade normalizes cerebrovascular autoregulation and reduces cerebral ischemia in spontaneously hypertensive rats. Stroke 31: 2478-2486.

Ormiston ML, Deng Y, Rundle N, Bendjelloul F, et al. (2013). A lymphocyte-dependent mode of action for imatinib mesylate in experimental pulmonary hypertension. Am. J. Pathol. 182: 1541-1551.

Park HS, Huh SH, Kim MS, Lee SH, et al. (2000). Nitric oxide negatively regulates c-Jun N-terminal kinase/stressactivated protein kinase by means of S-nitrosylation. Proc. Natl. Acad. Sci. U. S. A. 97: 14382-14387.

Paschen W, Gissel C, Linden T, Althausen S, et al. (1998). Activation of gadd153 expression through transient cerebral ischemia: evidence that ischemia causes endoplasmic reticulum dysfunction. Mol. Brain Res. 60: 115-122.

Rao RV, Peel A, Logvinova A, del Rio G, et al. (2002). Coupling endoplasmic reticulum stress to the cell death program: role of the ER chaperone GRP78. FEBS Lett. 514: 122-128.

Srinivasan K and Sharma SS (2012). 3-Bromo-7-nitroindazole attenuates brain ischemic injury in diabetic stroke via inhibition of endoplasmic reticulum stress pathway involving CHOP. Life Sci. 90: 154-160.

Sugino T, Nozaki K, Takagi Y, Hattori I, et al. (2000). Activation of mitogen-activated protein kinases after transient forebrain ischemia in gerbil hippocampus. J. Neurosci. 20: 4506-4514.

Tajiri S, Oyadomari S, Yano S, Morioka M, et al. (2004). Ischemia-induced neuronal cell death is mediated by the endoplasmic reticulum stress pathway involving CHOP. Cell Death Differ. 11: 403-415.

Tang F, Tang G, Xiang J, Dai Q, et al. (2002). The absence of NF-kappaB-mediated inhibition of c-Jun N-terminal kinase activation contributes to tumor necrosis factor alpha-induced apoptosis. Mol. Cell Biol. 22: 8571-8579.

Tripathi A and Sodhi A (2009). Growth hormone-induced production of cytokines in murine peritoneal macrophages in vitro: role of JAK/STAT, PI3K, PKC and MAP kinases. Immunobiol 214: 430-440.

Urano F, Wang X, Bertolotti A, Zhang Y, et al. (2000). Coupling of stress in the ER to activation of JNK protein kinases by transmembrane protein kinase IRE1. Science 287: 664-666.

Wang Q, Yin XH, Liu Y and Zhang GY (2011). K252a suppresses neuronal cells apoptosis through inhibiting the translocation of Bax to mitochondria induced by the MLK3/JNK signaling after transient global brain ischemia in rat hippocampal CA1 subregion. J. Recept. Signal Transduct. Res. 31: 307-313.

$\mathrm{Xu} \mathrm{W,} \mathrm{Liu} \mathrm{LH} \mathrm{and} \mathrm{Jia} \mathrm{YJ} \mathrm{(2012).} \mathrm{Protective} \mathrm{effect} \mathrm{of} \mathrm{endoplasmic} \mathrm{reticulum} \mathrm{stress} \mathrm{pretreatment} \mathrm{induced} \mathrm{by} \mathrm{2-deaeration}$ glucose on brain in ischemia/reperfusion rats. Progr. Anatom. Sci. 18: 362-369.

Zhang Q, Zhang G, Meng F and Tian H (2003). Biphasic activation of apoptosis signal-regulating kinase 1-stress-activated protein kinase 1-c-Jun $\mathrm{N}$-terminal protein kinase pathway is selectively mediated by $\mathrm{Ca}^{2+}$-permeable alpha-amino3-hydroxy-5-methyl-4-isoxazolepropionate receptors involving oxidative stress following brain ischemia in rat hippocampus. Neurosci. Lett. 337: 51-55. 